\title{
Lusioersily
}

\section{Classification effects on Motion-Onset Visual Evoked Potentials using commercially available video games}

Beveridge, R., Marshall, D., Wilson, S., \& Coyle, D. (2015). Classification effects on Motion-Onset Visual Evoked Potentials using commercially available video games. In Unknown Host Publication (pp. 28-37). IEEE. https://doi.org/10.1109/CGames.2015.7272958

Link to publication record in Ulster University Research Portal

\section{Published in:}

Unknown Host Publication

Publication Status:

Published (in print/issue): 01/01/2015

DOI:

10.1109/CGames.2015.7272958

\section{Document Version}

Publisher's PDF, also known as Version of record

\section{General rights}

Copyright for the publications made accessible via Ulster University's Research Portal is retained by the author(s) and / or other copyright owners and it is a condition of accessing these publications that users recognise and abide by the legal requirements associated with these rights.

\section{Take down policy}

The Research Portal is Ulster University's institutional repository that provides access to Ulster's research outputs. Every effort has been made to ensure that content in the Research Portal does not infringe any person's rights, or applicable UK laws. If you discover content in the Research Portal that you believe breaches copyright or violates any law, please contact pure-support@ulster.ac.uk. 


\title{
Classification Effects on Motion-Onset Visual Evoked Potentials using Commercially Available Video Games
}

\author{
Ryan Beveridge, David Marshall, Shane Wilson and Damien Coyle \\ Intelligent Systems Research Centre, Ulster University, Derry, Northern Ireland, U.K. \\ Beveridge-R@email.ulster.ac.uk, Marshall-D3@email.ulster.ac.uk, s.wilson@ulster.ac.uk and dh.coyle@ulster.ac.uk
}

\begin{abstract}
Motion Onset Visually Evoked Potentials (mVEPs) are elicited by visual stimuli that offer a more elegant, less fatiguing visual presentation than other stimuli used in visual evoked potentials (VEPs) studies. mVEP for use in brain computer interface (BCI) video gaming offer users a pleasant presentation environment to play video games. Modern, commercially available video games are a popular form of entertainment offering visually compelling, dynamic and graphically complex environments. However, most popular games exhibit visually fatiguing properties such as moving, flashing imagery and complex 3D shapes which may hinder accuracies of certain BCI paradigms. Because mVEP relies on motion visual stimulus rather than flashing imagery, it may be more apposite for use within complex game environments than other VEPs such as P300 and SSVEP. In this study we investigate the potential impact of varying levels of graphical fidelity from commercially available video games within an mVEP BCI control scheme. Building on a previous study, which investigated simplistic 3D based game levels, the current study investigates increased visual complexity in commercially available games from five different generations of gaming console and from different genres. We compared the visual effects of each of the five games on mVEP detection accuracy and found some of the more primitive properties of video games such as the use of primary colours, dynamic character movement, flashing imagery and the pace of the games have an influence on detection accuracies. These findings provide information relevant to design of a mVEP BCI game which is visually appealing to a wide range of users whilst maintaining $\mathrm{mVEP}$ accuracies.
\end{abstract}

Keywords - Brain-Computer Interface (BCI), Motion Onset Visually Evoked Potentials (mVEP), Electroencephalography (EEG), Gaming, Genre, 2D, 3D, Graphics, Console, Visual.

\section{INTRODUCTION}

Brain Computer Interfaces (BCI) are a method of computer control which allow users to operate a computer based system using only their neural activity thus bypassing the need for muscle control [1]. Traditional uses for this technology include control of assistive technologies such as prosthetic limbs [2], wheelchairs [3] and communication devices [4] for the physically impaired suffering from conditions such as Amyotrophic Lateral Sclerosis (ALS), brainstem stroke or spinal cord injury. In more recent times the rise in popularity and technological advancements in both graphics and hardware made to the gaming industry has given rise to the demand for new and novel control methods. Modern control methods such as Nintendo Wii in 2006 [5], Xbox Kinect in 2010 [6] and Oculus Rift in 2014 [7] have all provided the gaming industry with ways to make video games more social, intuitive and engaging to play. BCI as a control type for video games would provide players with a control method not seen before and has the potential of providing a movement free, novel and highly immersive gaming environment. BCI would also provide physically impaired users the opportunity to play video games as a form of entertainment due to its non-reliance on muscle control.

For BCI to progress as a viable compliment to traditional video games control and be accepted and adopted for commercial use, there are a number of impediments to overcome. One of the main technical issues restraining commercial use of BCI is latency. $\mathrm{BCI}$ latency refers to the time it takes for a BCI system to read, classify and convert the raw electroencephalography (EEG) signals into a useful command for a computer system to use. Existing BCI hardware also impedes widespread adoption of BCI technology due largely to cumbersome, expensive equipment and long setup times. However in recent years companies such as Emotiv [8] and NeuroSky [9] have developed wireless, dry electrode headsets which negate the requirement for wired interfaces to EEG equipment and conductive gel to be used during hardware setup. Advances such as these, along with consumer grade pricing ensure that BCI hardware will be an attractive technology in the future. Advances in EEG technology such as this helps to endorse BCI use in commercial video gaming by promoting ease of use and low prices. In recent years, advances in signal processing methods for BCI [10] have aided researchers in detecting relevant information more accurately from the noise 
contaminated and non-stationary neural signals produced by the human brain.

There are a number of brain signal features that can be used in BCIs. For example, P300 as used in studies such as [11][12] presents visual stimuli to the participant using flashing imagery. The P300 potential is a positive deflection seen in ongoing EEG signal with a latency of $250 \mathrm{~ms}$ to $500 \mathrm{~ms}$ post stimulus. The P300 stimulus is often elicited using the oddball paradigm which presents sequences of repetitive visual stimuli to the BCI user and by randomly presenting a divergent stimulus among the repetitive stimulus to the user, a $\mathrm{P} 300$ potential can be elicited. Although high accuracies $(>90 \%)$ can be achieved with its use and low requirement for user training, the inherent nature of flashing imagery can cause some visual fatigue for users of P300 VEPs. Steady State Visual Evoked Potentials (SSVEP) as used in [13][14] also makes use of flashing imagery to evoke visual potentials which are readable from the EEG. SSVEP works by presenting images to the user which flash at different but fixed frequencies. It is the frequency of the individual flashing items which produces recognisable properties in the ongoing EEG signal. SSVEP also provides high system accuracies $(>90 \%)$ and can be used without training but SSVEP can also cause visual fatigue to the user, so its use for video games may not be fully justified in an already visually rich environment.

mVEP uses moving imagery to elicit a response from the dorsal pathway of the brain [15][16] which provides a more visually pleasing and less fatiguing method of producing stimuli than other VEPs such as P300 and SSVEP. An mVEP response is composed of three main peaks post stimulus namely the P100 a positive peak observed $100 \mathrm{~ms}$ after stimulus, the N200 negative going peak $200 \mathrm{~ms}$ following stimulus presentation and the P300 positive going peak observed in the ongoing EEG signal around 240ms post stimulus. The brief motion of visual stimuli generates neural activations in the Medial Superior Temporal (MST) area of the brain which forms part of the cerebral cortex in the dorsal stream. The detection of motion takes place primarily in the Middle Temporal (MT) area of the brain.

In a previous study [17] we investigated how mVEP classification accuracy was affected by increasing visual complexity using a rudimentary $3 \mathrm{D}$ based game presentation that did not utilise high fidelity graphics. In this study however, we have used commercially available video games that cover five different generations of game consoles. The games chosen represent the state of the art of each games respective hardware technologies and era of graphical technology. Each of the games used were chosen according to their graphical maturity and gradually increased in graphic complexity. Also, the games presented cover a range of genres such as arcade, 2D platform, 3D platform, racing simulation and first person shooter to ensure adequate coverage of gameplay mechanics and dynamics [18][19].

Section 2 provides details on the methodology for the study. Section 3 is the data analysis section. Section 4 presents the results of the study. Section 5 provides a discussion and section 6 concludes the paper.

\section{METHODOLOGY}

\subsection{Paradigm}

The presentation environment used in the study was developed using the Unity 3D [20] game development engine. Five on screen virtual buttons, placed in a horizontal arrangement at the top of the computer screen form the mVEP game controller used in the study. In order to avoid visual distractions in the immediate area surrounding each mVEP button, the buttons were placed centrally within a plain white background acquiring approximately $13 \%$ of the total screen space. Each mVEP button subtends a visual field of $1.24^{\circ}$ length $\times 0.76^{\circ}$ height with the red coloured vertical moving line being $0.66^{\circ}$ in height.

Each mVEP button has a number (1 to 5) placed directly on top which differentiates them from each other. The subject identifies which button to concentrate on when the number of the current target button changes from black to red. The users' instructions were to focus their attention on the moving line of the currently active button (Fig. 1 shows the basic (no graphics) level with the ' 2 ' button as currently active while ' 2 ' is also the users current target (number highlighted red). A button activation constitutes one horizontal movement of the vertical red line from the left hand side to the right hand side of the vacant rectangle (lasting 140 milliseconds).

The timing protocol for this study followed closely to that of [17]. Each game level consisted of 300 trials. Each level lasted 540 seconds, during which, each mVEP button will have been activated 60 times. During each trial, each of the five mVEP buttons are active a total of five times. Each button is highlighted in turn starting from 1 through to 5 in a linear fashion. In order to avoid user habituation, each of the buttons are activated in random order. The Stimulus Onset Asynchrony (SOA) between each button activation is $200 \mathrm{~ms}$. The motion of the vertical line moving from right to left in each button 
lasts $140 \mathrm{~ms}$ and the time between each button activation is $60 \mathrm{~ms}$. A diagram of the timing protocol used is shown in (Fig. 2).

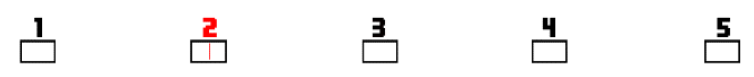

Figure 1. Level 1 - Plain white background. Buttons shown at top of the screen and no graphics presented.

300 Trails Total - 60 Trials per Stimulus - Each Stimulus Active 5 Times

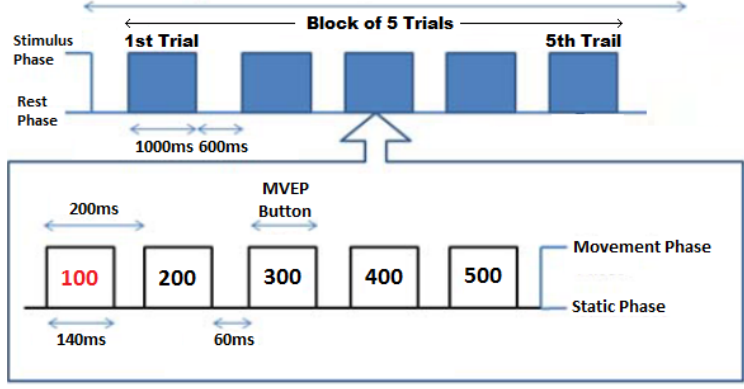

Figure 2. mVEP Timing protocol used in this study.

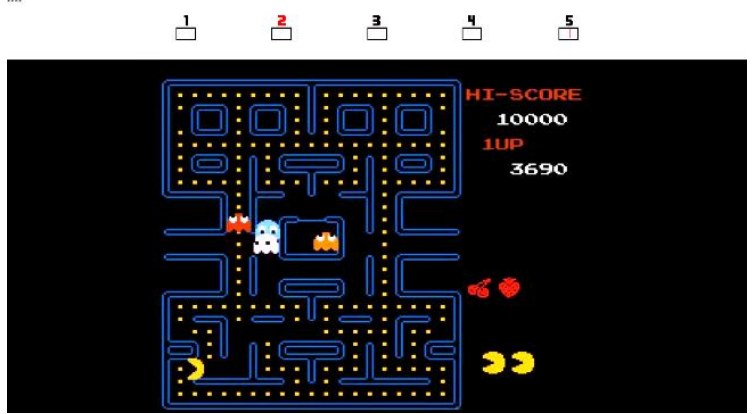

Figure 3. Level 2 - PacMan released on Nintendo (NES) Console (1984).

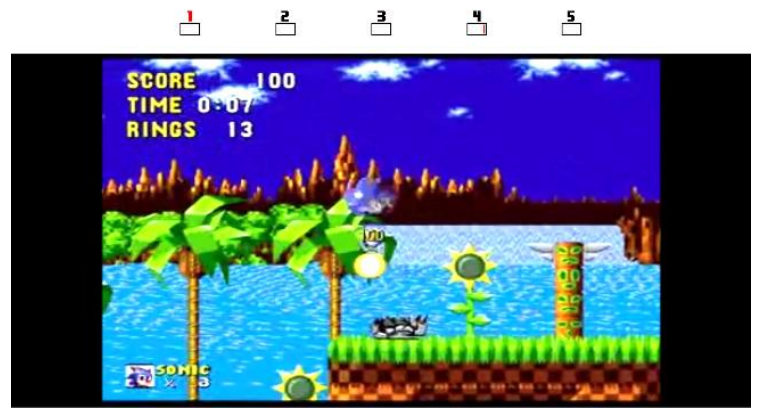

Figure 4. Level 3 - Sonic the Hedgehog released on the Sega Mega Drive (1991)

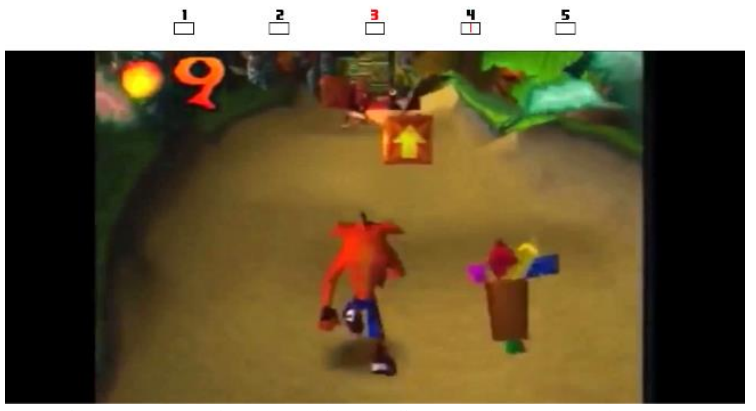

Figure 5. Level 4 - Crash Bandicoot released on the Sony Playstation (1996).

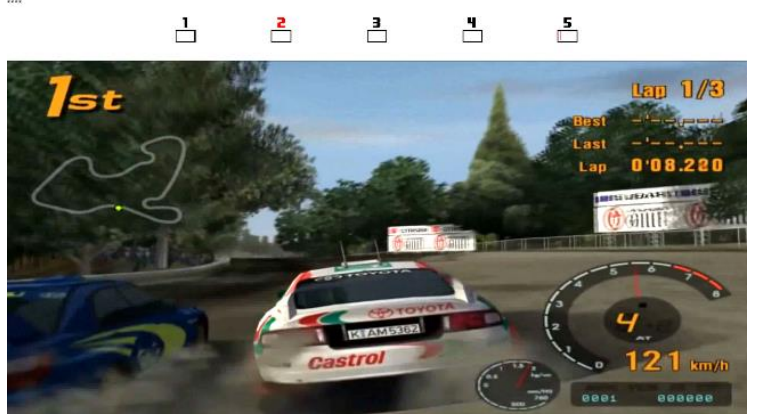

Figure 6. Level 5 - Gran Turismo 3 released on the Sony Playstation 2 (2001)
๖
몬
吕 5

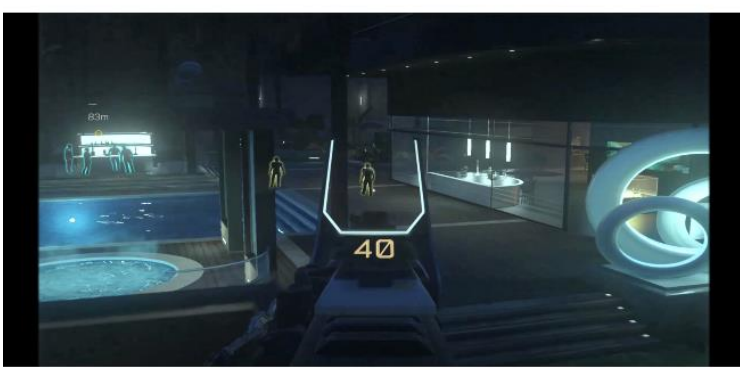

Figure 7. Level 6 - Call of Duty: Advanced Warfare as released on the Microsoft Xbox 360 (2014).

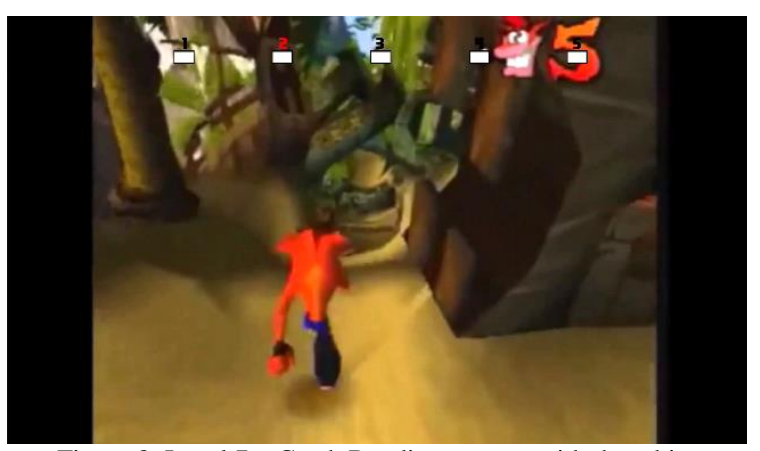

Figure 8. Level 7 - Crash Bandicoot game with the white background omitted from the MVEP button area.

The study consisted of seven different gameplay sessions presented to the subject as a video within the 
Unity 3D scene. Each of the games presented to the subject depicted typical commercially available games from varied generations of gaming consoles namely the $3^{\text {rd }}$ generation Nintendo Entertainment System (NES) [21], $4^{\text {th }}$ generation Sega Mega Drive $[22], 5^{\text {th }}$ generation Sony Playstation [23], $6^{\text {th }}$ generation Sony Playstation 2 [24] and the $7^{\text {th }}$ generation Microsoft Xbox 360 [25]. The games chosen represent the graphical achievements from the respective generation of games console. The games used in the study (in order of release date) and their respective game console were Pac-Man (Level 2) [26] - a fast paced 2D based arcade game featuring brightly coloured, flashing graphics. The goal of the player is to keep the main character constantly moving around a maze shaped world in order to collect food and avoid the enemy characters. The actual game world remains fairly static within the scene (Nintendo NES, 1984) (Fig. 3). Sonic the Hedgehog (Level 3) [27] - a very fast paced 2D platform game featuring brightly coloured game background and very fast paced character. The goal of the player is to navigate the main character through the game world by jumping over obstacles, collecting rings and avoiding or killing enemy characters. The world within the game is very dynamic and constantly changes as the level progresses (Sega Mega Drive, 1991) (Fig. 4). Crash Bandicoot (Level 4) [28] - a very fast paced $3 \mathrm{D}$ based platform game which features a brightly coloured 3D game world and fast paced character movements. The goal of the player is to jump over and smash obstacles, avoid and kill enemy characters and collect food. The game world is very dynamic and constantly changes throughout the level (Sony Playstation, 1996) (Fig. 5). Gran Turismo 3 (Level 5) [29] - a fast paced 3D racing simulation game featuring constantly moving but steadily paced high fidelity $3 \mathrm{D}$ graphics. The player controls a series of realistic cars around real world racing tracks (Sony Playstation 2, 2001) (Fig. 6). Call of Duty: Advanced Warfare (Level 6) [30] - a fast paced 3D based first person shooter which features realistic, high fidelity graphics and the goal of the player is to control a soldier around a futuristic but realistic game world (Microsoft Xbox 360, 2014) (Fig. 7). In order to compare each game against a more rudimentary graphical presentation, a level which contained no graphics was also introduced to the subjects which comprised a plain white background (Level 1) (Fig. 1). To assess and compare if the moving graphics in the immediate area surrounding the buttons affected the mVEP accuracy, we added a further level within the session which omitted the plain white background surrounding the buttons where the buttons became overlaid onto the games graphics. For this level we chose a game that is considered by the average game complexity among the games namely level 4 (Level 7) (Fig. 8). To assess and rule out user fatigue for each of the game levels during the course of the session, the games were presented to the subjects in a randomly ordered fashion. To keep focus on the accuracy of the mVEP paradigm against the graphical properties of the games, the task of the user was only to mentally count the number of times the highlighted button was activated, therefore they had no control over the game and no real-time feedback on performance was provided.

\subsection{Data Acquisition}

Ten healthy male subjects took part in this study with an age range between 20 and 38 years (average 26 years). Four of these subjects had previous BCI experience using $\mathrm{mVEP}$ and the remaining six were BCI naive. All ten subjects took part in a single recording session, which comprised of watching the seven video game presentations in random order with a short five minute break between each one. Electrode placement was strategically chosen over occipital areas using a 12 channel montage (Fig. 9) according to the international 10-20 system of electrode placement [31]. The left mastoid acted as ground and $\mathrm{FPz}$ as the reference voltage. Recording took place in a darkened, electrostatically shielded and acoustically insulated room. Participants were seated on a comfortable chair at a distance of $50 \mathrm{~cm}$ in front of an LCD computer monitor $56 \mathrm{~cm}$ (Width $47.7 \mathrm{~cm}$ and Height $29.8 \mathrm{~cm}$ ) in size. The refresh rate of the monitor was $60 \mathrm{~Hz}$ and the resolution set to $1680 \times 1050$ pixels.

EEG data was collected using a g.BSamp amplifier [32] with $50 \mathrm{~Hz}$ notch filter to eliminate powerline noise interference. A g.Gammasys active electrode system [33], connected to an Easycap electrode cap [34] was used and Matlab Simulink [35] analysed the data. As Unity 3D presented each visual cue to the user, a stimulus identifier relating to each mVEP stimulus along with timing information were sent to Simulink from Unity 3D using the User Datagram Protocol (UDP) transmission convention. The stimulus trigger information and EEG signals are co-registered in Simulink. 


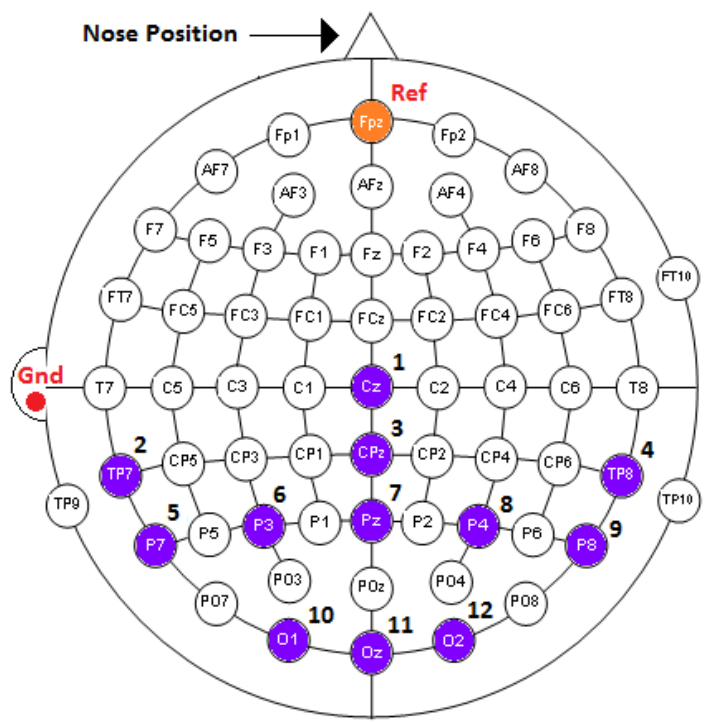

Figure 9. 12 Channel montage used in the study with FPz as reference voltage and left mastoid as ground.

\section{DATA ANALYSIS}

\subsection{Data pre-processing Methods}

As each of the five stimuli (mVEP buttons) were a target for 60 trials for each game level, we were able to record a total of 300 trials per level from each subject. Data epochs were derived in association with each motion onset stimulus, beginning 200ms prior to the motion onset and lasting for $1200 \mathrm{~ms}$. All single trials were baseline corrected with respect to the mean voltage over the $200 \mathrm{~ms}$ preceding motion onset. Data were digitally filtered using a low-pass Butterworth filter (order 5, with cut-off at $10 \mathrm{~Hz}$ ) and subsequently resampled at $20 \mathrm{~Hz}$. Features were extracted between the $100 \mathrm{~ms}$ and $500 \mathrm{~ms}$ epoch post stimulus which normally contains the most reactive mVEP components e.g. N200, P300 and N400. This yields nine features for each channel. Data were averaged over five trials yielding twelve feature vectors per stimulus for each level. Data were initially split into target vs. non-target where for each non-target feature vector five randomly selected nontarget trials were used.

mVEP is time locked and phase locked to the motion onset stimulus therefore mVEP induced from the motion stimuli could be obtained through the above simple averaging procedure [15].

\subsection{Channel Selection}

A Linear Discriminant Analysis (LDA) classifier was trained to discriminate target vs. non target feature vectors extracted from single channels in a Leave One Out (LOO) cross validation on $50 \%$ of the data (the remaining 50\% was held out for final testing). For each of the twelve channels the average LOO classification accuracy (LOO-CA) was determined and channels were ranked by accuracy. The most commonly highest ranked channels across all subjects consisted of O1, P7 and TP7. The top three ranked channels were concatenated to form a new feature vector (27 features per vector) and a further LOO cross validation was performed. The results of this are reported as LOO-CA3. A single trial test of target vs. non target is also applied on the training data (Target vs. Non Target - Single Trial).

\section{3. mVEP Classification - 5 Class}

Using all the training data ( $50 \%$ of data) a new LDA classifier is produced to classify target vs. non target data. To classify individual symbols in a single trial test each feature vector associated with each stimulus in a trial is classified as either target or nontarget. The LDA classifier produced a distance value, $D$, reflecting the distance from the hyper plane separating target and non-target features $(D>0$ for target and $D<0$ for non-target). The vector that produces the maximum distance value is selected as the classified stimulus (in some cases non-target data produces a $D>0$, however the value of $D$ is normally maximal among the target stimulus i.e., the stimulus on which the user is focused). Single trial results for five class are reported for the training data and then the setup is applied on the remaining $50 \%$ of the data, unseen testing data.

Offline analysis was performed using customised MATLAB code along with the BioSig [36] and LIBSVM [37] toolboxes.

\section{RESULTS}

\subsection{Offline Testing}

Data from all ten subjects were analysed for each of the 5 game levels with the addition of the training level and the Crash Bandicoot game with the white background omitted from the button controller area. Four methods are used to analyse the subjects data namely, LOO-CA3 (test 1), target vs. non target single trial (training) (test 2), single trial 5 class (training) (test 3) and single trial 5 class (testing) (test 4).

Fig. 10 shows the average test 1 result for all ten subjects across the seven game levels. The graph shows a linear decline in accuracy for the first four game levels $(76.1 \%, 74.5,74.5 \%$ and $71.6 \%)$ respectively. Level 5 shows an increase in accuracy $(78.7 \%)$ leaving it the game with the best overall mVEP accuracy of all levels. By levels six and seven (73.6\% and $72.2 \%$ ) respectively, the MVEP accuracy declines linearly from level 5 . The game level with 
the worst accuracy was level 4 (71.6\%). An Analysis of Variance ANOVA test was conducted between the best and worst performing game levels (level 5 and level 4 respectively) and returned a value of $p=0.01$, suggesting the differences in accuracy are statistically significant. An ANOVA test between level 4 and level 7 (Crash Bandicoot with and without white button background) revealed a result of $p=0.69$ revealing the difference between these two levels are not statistically significant. A further ANOVA test was conducted taking into account all game levels and returned a value of $p=0.18$ suggesting that the difference in accuracy for all levels are not statistically significant.

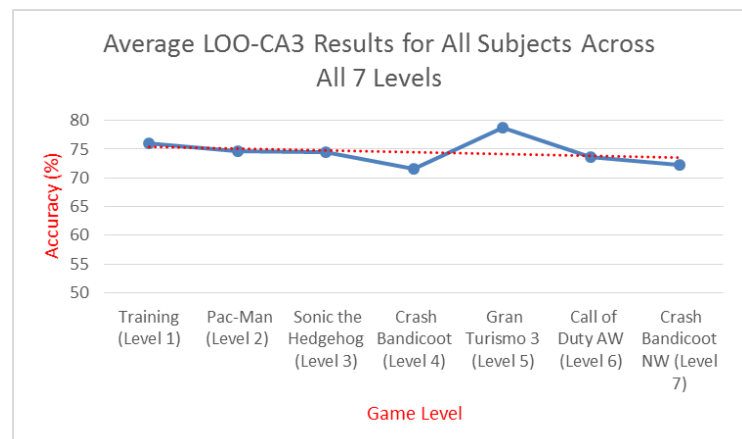

Figure 10. Graph to show the average LOO-CA3 (test 1) accuracy for all ten subjects for all seven game levels.

Fig. 11 shows the graph of the test 2 analysis which shows similar trends of test 1 analysis above. In this graph we can see an increase in level $2(80.6 \%)$ over level $1(79.4 \%)$ but this drops again by level 3 (78.2\%). Again, level 5 produces the best accuracy $(83.6 \%)$ and level 4 produces the worst accuracy $(72.8 \%)$ of all levels. Also to note in this graph, the most graphically complex game - level 6 produces the second best accuracy achievement (80\%). Level 7 obtained higher classification results $(75.1 \%)$ than the level $4(72.8 \%)$ suggesting that the moving game objects surrounding the mVEP buttons did not adversely affect the accuracy of the system. ANOVA results comparing the best (level 5) and worst (level 4) game presentations for test 2 return a value of $p=0.05$ showing the statistical significance of the results. ANOVA comparing the level 4 and level 7 provide a value of $p=0.65$ showing that the results are not statistically significant. Taking all levels into account, ANOVA results for test 2 returned a value of $p=0.45$.

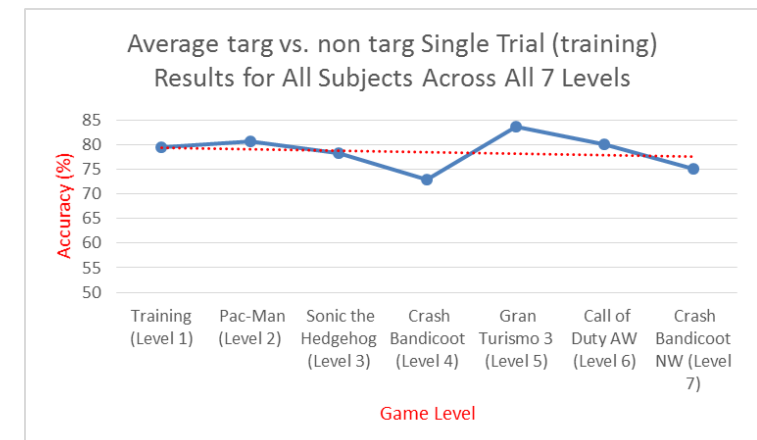

Figure 11. Graph to show the mean Target vs. non Target Single Trial (training) (test 2) accuracy across all game presentations.

Fig. 12 shows the results for the test 3 analysis. As can be seen a drop in mVEP accuracy from level $1(79.2 \%)$ to level $2(78.5 \%)$ and again at level 3 $(70.8 \%)$. Level $4(70.8 \%)$ produced the same results as the level 3 with these levels having the worst overall accuracies. Once again, level 5 obtained the highest accuracy $(83.5 \%)$ of all levels and level 6 following with the second highest accuracy (79.6\%). As with the previous analysis tests, these results also show level 7 with no white background surrounding the mVEP buttons produced a higher accuracy $(74.6 \%)$ than level 4 with the white background $(70.8 \%)$. ANOVA results between level 4 and level 5 game levels return a result of $p=0.03$ indicating the results are statistically significant. ANOVA between the two levels 4 and level 7 return a value $p=0.48$, furthering the point that the results are insignificant. ANOVA using all game levels provide a value of $p=0.31$, again suggesting that the difference in $\mathrm{mVEP}$ accuracies using all games are not significant.

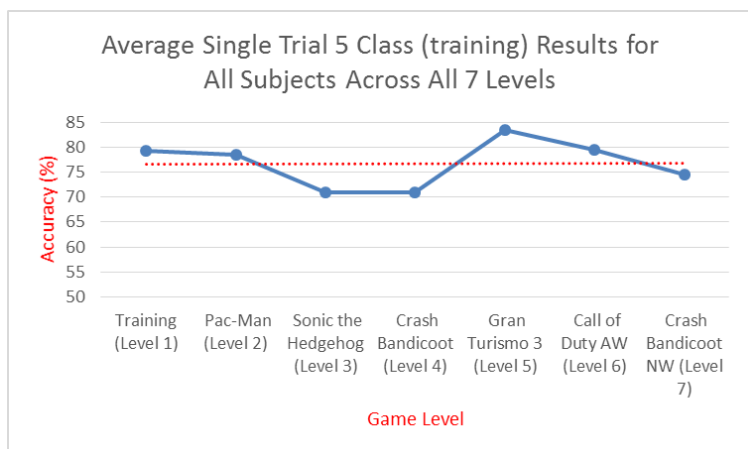

Figure 12. Graph to show the mean Single Trial 5 Class (training) (test 3 ) accuracy across all game presentations.

Test 4 is the final analysis test to be conducted on the subjects' data. Fig. 13 shows the results graph and this time the greatest accuracy can be seen in level $2(63.6 \%)$ which performed slightly higher than the level $5(62.8 \%)$ which performed second best overall. 
Using this analysis test, level 7 (54\%) obtained the worst accuracy of all the game levels.

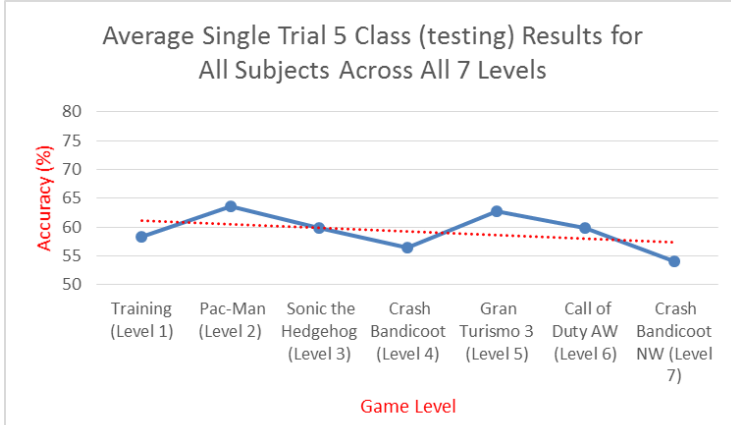

Figure 13. Graph to show the mean Single Trial 5 Class (testing) (test 4) accuracy across all game presentations.

The results presented here using the four analysis tests show that the Gran Turismo 3 game level consistently obtained the greatest accuracy $(78.7 \%, 83.6 \%$ and $83.5 \%)$ respectively for analysis tests 1, 2, and 3. The Pac-Man level claimed the greatest accuracy $(63.6 \%)$ using the final analysis test narrowly having the advantage over the Gran Turismo 3 level (62.8\%). The Crash Bandicoot level showed consistently low results compared all other levels in tests 1, 2 and 3 (congruent in test 3 with the Sonic the Hedgehog level) and performed second worst overall in the results for test 4 . The results for test 4 were lower than test 1,2 and 3 as the system is required to test on unseen training data (final $50 \%$ of data is held out for final system testing). The lower accuracies could be attributed to the EEG data being contaminated with artefacts such as eye blinks, body movements or mental fatigue.

\subsection{Individual Subject Performances}

TABLE I. TABLE TO SHOW RESULTS FOR INDIVIDUAL SUBJECTS ACROSS ALL ANALYSIS METHODS (\%).

\begin{tabular}{|c|c|c|c|c|}
\cline { 2 - 5 } \multicolumn{1}{c|}{} & \multicolumn{3}{c|}{ Training Assessment } & Testing \\
\cline { 2 - 5 } \multicolumn{1}{c|}{} & \multicolumn{2}{c|}{ Target vs Non Target } & Five Class & Five Class \\
\cline { 2 - 5 } \multicolumn{1}{c|}{} & L00-CA3 & Single Trial & Single Trial & Single Trial \\
\hline S1 & 71.6 & 69.8 & 68.5 & 56.9 \\
\hline S2 & 79.0 & 86.3 & 84.7 & 69.6 \\
\hline S3 & $\mathbf{8 0 . 0}$ & $\mathbf{8 9 . 9}$ & $\mathbf{8 8 . 0}$ & $\mathbf{7 0 . 7}$ \\
\hline S4 & 76.6 & 85.3 & 82.7 & 63.6 \\
\hline S5 & 70.1 & $\mathbf{6 8 . 8}$ & $\mathbf{6 8 . 2}$ & 46.5 \\
\hline S6 & 76.5 & 84.0 & 83.6 & 67.0 \\
\hline S7 & 71.4 & 72.2 & 72.4 & 49.7 \\
\hline S8 & 74.8 & 76.7 & 77.3 & 60.6 \\
\hline S9 & 75.1 & 82.4 & 83.7 & 64.6 \\
\hline S10 & $\mathbf{6 9 . 7}$ & 70.0 & 68.6 & $\mathbf{4 4 . 0}$ \\
\hline Average & 74.5 & 78.5 & 77.8 & 59.3 \\
\hline
\end{tabular}

In table I above, we can see the overall results achieved from all ten subjects. Subject S3 performed best throughout all four analysis tests (bold and italic text) achieving $80 \%, 89.9 \%, 88 \%$ and $70.7 \%$ respectively. Subject S10 performed worse out of all ten subjects using analysis test 1 and test 4 achieving $69.7 \%$ and $44 \%$ respectively (bold underlined text) and subject S5 performed worse overall for the test 2 and test 3 analysis achieving $68.8 \%$ and $68.2 \%$ respectively.

We can see in the table that participants consistently obtained accuracies of $>70 \%$ for analysis test 1 (mean $74.5 \%$ ), test 2 (mean $78.5 \%$ ), test 3 (mean $77.8 \%$ ). Analysis test 4 obtained accuracies between $44 \%$ and $70.7 \%$ (mean $59.3 \%$ ).

\section{DISCUSSION}

Advantages such as low visual fatigue, high system accuracy and low training requirement renders the mVEP paradigm a more suitable visual stimulus presentation for use in complex game environments than other VEP based BCI paradigms such as P300 and SSVEP [18]. In this study, following on from a previous study [17] we have investigated commercially available games from five different generations of game console in an attempt to identify suitable graphics and genre for use within a game designed using the mVEP paradigm as a control method. By presenting the various graphically complex games to the participant in random order, we were able to better rule out user fatigue during the course of each session. This enabled a fair comparison of each game level regardless of subject fatigue or other environmental factors e.g., electrode gel drying and electrode movement.

Presenting the basic (no graphics) training level to the subjects produced a varied result ranging from $2^{\text {nd }}$ in analysis test $1,3^{\text {rd }}$ in test $3,4^{\text {th }}$ in analysis test 2 and $5^{\text {th }}$ in analysis test 4 . We can see from the results that even though the basic training level presented no visual distractions to the subject, the varied results suggest that the lack of graphical content in the level offered no advantage to the mVEP paradigm used. One reason for this finding may be that most of the subjects found the training level mundane due to the static presentation environment and high luminosity of the plain white background.

Also, the Crash Bandicoot game level with the white background removed from the mVEP button area produced greater accuracies $(72.2 \%, 75.1 \%$ and $74.6 \%$ ) for tests 1,2 and 3 respectively compared to the same game level with white background which achieved the results $(71.6 \%, 72.8 \%$ and $70.8 \%)$ for tests 1,2 and 3 respectively. This trend was noticed in all of the analysis tests except test $4(54.0 \%$ for Crash Bandicoot No White vs. $56.4 \%$ for Crash Bandicoot with the white background). It may be that the greater accuracy for the game with no white 
background surrounding the mVEP buttons is noticed because the participant becomes accustomed (trained) to the white background on the mVEP button control area in all other game levels. Suddenly, by omitting the white background the subject may realise the novelty of the game environment surrounding the MVEP buttons and so not only does it become more interesting, it is also required that the subject needs to concentrate on the buttons in a more focused manner due to the extra distractions in the controller area.

The graphical complexity contained in the Gran Turismo 3 game level encompasses the second most complex graphics of the game levels tested in this study. However, even though the graphics are technically more advanced and realistic than e.g., Pac-Man, Sonic the Hedgehog and Crash Bandicoot, we can see clearly higher mVEP system accuracies for Gran Turismo 3. After further analysis we have concluded that the uniformly paced gameplay and consistent background of Gran Turismo 3 created an interesting and compelling visual environment with minimal visual distractions. These factors contributed to the greater mVEP accuracy achieved by participants with the Gran Turismo 3 game level. In comparison, the Pac-Man game level presents an environment which contains flashing imagery and game objects represented by bright primary colours with erratic movement. Furthermore, the Sonic the Hedgehog game level contains a very non-static scenery with a constantly moving game character and the use of primary colours, flashing imagery and variable, constantly changing speed which all contribute to the advanced workload of the participants' visual processing area of the brain. The Crash Bandicoot level produced the worst overall results across all four analysis tests. In this game level the protagonist depicted by a brightly coloured, rapidly moving character who constantly changes from jumping, bouncing, spinning and falling presents a highly dynamic game environment to the subjects which furthers the point that the faster paced, brightly coloured gameplay scenarios faced by the participants contribute to a lower mVEP classification accuracy.

The most graphically complex game used in this study was the Call of Duty: Advanced Warfare game level which represents the current state of graphics in today's gaming industry. In this game level we also see a varied result in the accuracies: $5^{\text {th }}$ in analysis test $1,2^{\text {nd }}$ in analysis test $3,3^{\text {rd }}$ in analysis test 2 and $3^{\text {rd }}$ in analysis test 4 . The results suggest that using the latest graphical techniques such as those employed in Call of Duty: Advanced Warfare may be feasible for an mVEP controlled BCI game without radically depleting the system classification accuracies.

\subsection{Limitations}

Ten subjects were tested for this study. Perhaps if more subjects were tested, the ANOVA analysis may have shown the significance of differences between the complexity of each level. The games presentation used for the study were videos played to each subject of commercially available video games. Due to the games presentation being shown via video and the mVEP training environment used, the subjects had no control over the gameplay and thus were not provided with feedback. The lack of feedback may have affected the accuracies achieved by the subjects as the task was only to concentrate on the mVEP button area of the screen and most subjects reported that they felt some feedback would have maintained their attention better.

We selected only one game (Crash Bandicoot) out of the five to present the mVEP button area without the white background. If we had presented the mVEP button area to the subjects without the white background using all the game levels, it would have allowed a better insight into the performance gain or loss of each type of game using this method of controller. We did not test the no-white background on all games due to time constraints on the session, however testing these two controller methods over two sessions (one for white background and one for no white background) may provide clearer results.

\section{CONCLUSION}

The results of this study suggest that graphical complexity alone does not degrade the mVEP accuracy using the paradigm described above but it may be the more primitive properties of video games such as primary colours, dynamic character movement, flashing imagery and pace which degrade results. The results do suggest that certain graphics and genres may be more suited to $\mathrm{mVEP}$ paradigms than others. The results from this study will be considered when designing an online mVEP based BCI game which provides high fidelity graphics in a par with those of commercially available games.

\section{FUTURE WORK}

Integration using an Oculus Rift virtual reality device with this mVEP BCI paradigm is a project currently in development. The use of such a 
technology as the Oculus Rift may offer increased levels of immersion for the participants and will provide a play environment where the onscreen mVEP buttons remain statically located on the display area, thus helping concentration on the controller area.

A further study using the mVEP paradigm presented here whilst using an eye tracking device such as the Tobii EyeX [38] may also be conducted in the future which will help to distinguish artefact noises from eye movements. Eye tracking technology may also be used as a compliment to game controls as in a hybrid BCI between mVEP and eye tracking technology.

The development of an online mVEP based BCI game is also proposed and will offer participants real time feedback.

\section{REFERENCES}

[1] J. R. Wolpaw, N. Birbaumer, D. J. McFarland, G. Pfurtscheller, and T. M. Vaughan, "Brain-computer interfaces for communication and control.," Clin. Neurophysiol., vol. 113, no. 6, pp. 767-91, Jun. 2002.

[2] D. Nishikawa, W. Yu, H. Yokoi, and Y. Kakazu, "Online learning method for EMG prosthetic hand control," Electron. Commun. Japan (Part III Fundam. Electron. Sci., vol. 84, no. 10, pp. 35-46, Oct. 2001.

[3] Y. Punsawad and Y. Wongsawat, "Hybrid SSVEPmotion visual stimulus based BCI system for intelligent wheelchair.," Conf. Proc. IEEE Eng. Med. Biol. Soc., vol. 2013, pp. 7416-9, Jul. 2013.

[4] B. Hong, F. Guo, T. Liu, X. Gao, and S. Gao, "N200speller using motion-onset visual response.," Clin. Neurophysiol., vol. 120, no. 9, pp. 1658-66, Sep. 2009.

[5] "Nintendo Wii." [Online]. Available: https://www.nintendo.co.uk/Wii/Wii-94559.html. [Accessed: 01-Oct-2014].

[6] "Xbox 360 Kinect (Microsoft)." [Online]. Available: http://www.xbox.com/en-gb/kinect. [Accessed: 07-Oct2014].

[7] “Oculus Rift,” 2015. [Online]. Available: http://www.oculus.com/. [Accessed: 01-Oct-2014].

[8] "Emotiv EPOC.” [Online]. Available: http://emotiv.com/. [Accessed: 05-Mar-2015].

[9] "NeuroSky MindWave.” [Online]. Available: http://store.neurosky.com/products/mindwave-mobile. [Accessed: 05-May-2015].
[10] D. Coyle, J. Garcia, A. R. Satti, and T. M. McGinnity, "EEG-based continuous control of a game using a 3 channel motor imagery BCI: BCI game," 2011 IEEE Symp. Comput. Intell. Cogn. Algorithms, Mind, Brain, pp. 1-7, Apr. 2011.

[11] G. Pires, M. Torres, N. Casaleiro, U. Nunes, and M. Castelo-Branco, "Playing Tetris with non-invasive BCI,” 2011 IEEE 1st Int. Conf. Serious Games Appl. Heal., pp. 1-6, Nov. 2011.

[12] A. Finke, A. Lenhardt, and H. Ritter, "The MindGame: a P300-based brain-computer interface game.," Neural Netw., vol. 22, no. 9, pp. 1329-33, Nov. 2009.

[13] P. Martinez, H. Bakardjian, and A. Cichocki, "Fully online multicommand brain-computer interface with visual neurofeedback using SSVEP paradigm.,' Comput. Intell. Neurosci., vol. 2007, no. i, p. 94561 Jan. 2007.

[14] E. C. Lalor, S. P. Kelly, C. Finucane, R. Burke, R Smith, R. B. Reilly, and G. McDarby, "Steady-State VEP-Based Brain-Computer Interface Control in an Immersive 3D Gaming Environment," EURASIP J. Adv. Signal Process., vol. 2005, no. 19, pp. 3156-3164, 2005.

[15] F. Guo, B. Hong, X. Gao, and S. Gao, "A braincomputer interface using motion-onset visual evoked potential.," J. Neural Eng., vol. 5, no. 4, pp. 477-85, Dec. 2008

[16] D. Marshall, S. Wilson, and D. Coyle, "Motion-Onset Visual Evoked Potentials for Gaming: A pilot study," 24th IET Irish Signals Syst. Conf. (ISSC 2013), pp. 56$56,2013$.

[17] R. Beveridge, D. Marshall, S. Wilson, and D. Coyle "3D Game Graphic Complexity Effects on MotionOnset Visual Evoked Potentials," in CGAT 2015, 2015, pp. 139-147.

[18] D. Marshall, D. Coyle, S. Member, S. Wilson, and M. Callaghan, "Games , Gameplay, and BCI : The State of the Art," vol. 5, no. 2, pp. 82-99, 2013.

[19] D. Marshall, S. Wilson, and D. Coyle, "Motion-Onse Visual Evoked Potentials for Gaming,” pp. 155-164, 1901.

"Nintendo UK." [Online]. Available: https://www.nintendo.co.uk/index.html. [Accessed: 22Apr-2015]

http://www.sega.co.uk/. [Accessed: 22-Apr-2015]. 
[23] "Playstation," 1995. [Online]. Available: http://uk.playstation.com/. [Accessed: 29-May-2014].

[24] "Playstation." [Online]. Available: https://www.playstation.com/en-gb/. [Accessed: 22Apr-2015].

[25] "Xbox." [Online]. Available: http://www.xbox.com/en$\mathrm{gb} /$. [Accessed: 22-Apr-2015].

[26] "PACMAN.” Namco, 1980.

[27] "Sonic the Hedgehog." [Online]. Available: http://www.sonicthehedgehog.com/en/. [Accessed: 22Apr-2015].

[28] "Crash Bandicoot." [Online]. Available: https://www.playstation.com/en-gb/games/crashbandicoot-ps3/. [Accessed: 18-May-2015].

[29] “Gran Turismo.” [Online]. Available: http://www.granturismo.com/gb/. [Accessed: 22-Apr-2015].

[30] "Call of Duty: Advanced Warfare." [Online]. Available: https://www.callofduty.com/uk/en/advancedwarfare. [Accessed: 22-Apr-2015].

[31] "International 10-20 System." [Online]. Available: http://en.wikipedia.org/wiki/10-20_system_(EEG). [Accessed: 29-May-2014].
[32] “g.tec - g.BSamp Biosignal Amplifier," 2014. [Online]. Available: http://www.gtec.at/Products/Hardware-andAccessories/g.BSamp-Specs-Features. [Accessed: 29May-2014].

[33] “g.Tec - g.GAMMAbox," 2014. [Online]. Available: http://www.gtec.at/Products/Electrodes-andSensors/g.GAMMAsys-Specs-Features. [Accessed: 29May-2014].

[34] "Easycap EEG Cap.”[Online]. Available: http://www.easycap.de/easycap/. [Accessed: 29-May2014].

[35] “MATLAB.” MathWorks, 2014.

[36] "BioSig Toolbox." [Online]. Available: http://biosig.sourceforge.net/. [Accessed: 06-Jan-2014].

[37] "LIBSVM Toolbox." [Online]. Available: http://www.csie.ntu.edu.tw/ cjlin/libsvm/. [Accessed: 06-Jan-2014].

[38] “Tobii EyeX.” [Online]. Available: http://www.tobii.com/en/eye-experience/eyex/. [Accessed: 18-Jun-2015]. 